OPEN ACCESS

Edited by:

Marco Borghesi,

University of Genoa, Italy

Reviewed by:

Andrea Mari,

University of Florence, Italy

Leena Latonen,

University of Eastern Finland, Finland

*Correspondence:

Zhize Wang

wangzhize@zju.edu.cn

${ }^{\dagger}$ These authors have contributed equally to this work

FThese authors share senior authorship

Specialty section: This article was submitted to Genitourinary Oncology, a section of the journal

Frontiers in Oncology

Received: 15 June 2020 Accepted: 07 October 2020 Published: 30 November 2020

Citation:

Wang Z, Shen H, Ma N, Li Q, Mao Y, Wang $C$ and Xie $L$ (2020) The

Prognostic Value of Androgen

Receptor Splice Variant 7 in Castration-Resistant Prostate Cancer Treated With Novel Hormonal Therapy or Chemotherapy: A Systematic Review and Meta-analysis.

Front. Oncol. 10:572590. doi: 10.3389/fonc.2020.572590

\section{The Prognostic Value of} Androgen Receptor Splice Variant 7 in Castration-Resistant Prostate Cancer Treated With Novel Hormonal Therapy or Chemotherapy: A Systematic
Review and Meta-analysis

\author{
Zhize Wang ${ }^{1 * t \neq}$, Haixiang Shen ${ }^{1 \dagger}$, Nieying $\mathrm{Ma}^{2}$, Qinchen $\mathrm{Li}^{1}$, Yeqing Mao ${ }^{1}$, \\ Chaojun Wang ${ }^{1 \neq}$ and Liping Xie ${ }^{1 \neq}$ \\ ${ }^{1}$ Department of Urology, The First Affiliated Hospital, Zhejiang University School of Medicine, Hangzhou, China, ${ }^{2}$ Key \\ laboratory of Reproductive Genetic (Ministry of Education) and Department of Reproductive Endocrinology, Women's \\ Hospital, Zhejiang University School of Medicine, Hangzhou, China
}

Purpose: This study aimed to evaluate the prognostic role of AR-V7 in terms of prostatespecific antigen (PSA) response, progression-free survival (PFS), and overall survival (OS) in CRPC patients treated with novel hormonal therapy (NHT) (Abiraterone and Enzalutamide) or taxane-based chemotherapy (Docetaxel and Cabazitaxel).

Methods: A comprehensive literature search was conducted on PubMed, Embase, and the Web of Science from inception to February 2020. Studies focusing on the prognostic values of AR-V7 in CRPC patients treated with $\mathrm{NHT}$ or chemotherapy were included in our meta-analysis. The OS and PFS were analyzed based on Hazard ratios (HRs) and 95\% confidence intervals (Cls). Furthermore, Odds ratios (ORs) and 95\% Cls were summarized for the AR-V7 conversion after treatment and the PSA response.

Results: The AR-V7 positive proportion increased significantly after NHT treatment (OR 2.56, 95\% Cl 1.51-4.32, P<0.001), however, it declined after chemotherapy (OR 0.51, 95\% Cl 0.28-0.93, $\mathrm{P}=0.003$ ). AR-V7-positive patients showed a significantly decreased PSA response rate after NHT (OR 0.13, 95\% Cl 0.09-0.19, $\mathrm{P}<0.001$ ) but not statistically significant for chemotherapy (OR 0.63, 95\% Cl 0.40-1.01, P=0.06). Notably, PFS (HR 3.56, 95\% Cl 2.53-5.01, $\mathrm{P}<0.001$ ) and OS (HR 4.47, 95\% Cl 3.03-6.59, $\mathrm{P}<0.001$ ) were worse in AR-V7-positive ttreated with NHT. Similarly, AR-V7 positivity correlated with poor prognosis after chemotherapy as evidenced by shorter OS (HR 1.98, 95\% Cl 1.48-2.66, $\mathrm{P}<0.001)$ and a significantly shorter PFS (HR 1.35, 95\% Cl 0.97-1.87, $\mathrm{P}=0.07)$.

Conclusion: NHT treatment increased $A R-V 7$ positive proportion whereas chemotherapy decreased it. Moreover, AR-V7 positivity correlated with lower PSA 
response, poorer PFS, and OS in CRPC treated with $\mathrm{NHT}$, and shorter OS in patients receiving chemotherapy.

Keywords: androgen receptor splicing variant 7, novel hormonal therapy, chemotherapy, prostate cancer, predictor, survival

\section{INTRODUCTION}

Taxane-based chemotherapy (Docetaxel and Cabazitaxel) and novel hormonal therapy (NHT) such as Enzalutamide and Abiraterone have been proven to improve overall survival (OS) and possess favorable safety profiles in metastatic castrationresistant prostate cancer (mCRPC) $(1,2)$. The prognosis of most mCRPC patients is poor which calls for development of effective biomarkers to predict treatment outcomes and choices $(3,4)$. The constitutively active AR splice variants lacking the androgen ligand-binding domain contribute to NHT resistance $(5,6)$. Accumulating evidence suggests that Abiraterone and Enzalutamide are not effective in AR-V7-positive patients (79). However, AR-V7-positive patients showed a better response to taxane chemotherapy $(8,10,11)$. Therefore, AR-V7 may guide treatment decisions for CRPC patients (12).

Several studies have reported that AR-V7 is unable to predict the effectiveness of NHT in CRPC $(13,14)$. Moreover, AR-V7 positivity has been associated with unfavorable baseline characteristics and a higher tumor burden $(10,15)$. AR-V7 mediates taxane resistance via several mechanisms. Previously, high expression of AR-V7 was linked to NHT resistance, but its expression level declined after chemotherapy $(7,8,16-21)$. In recent years, the prognostic value and clinical utility of AR-V7 in NHT and chemotherapy are deeply investigated. Promising data support AR-V7 as a treatment selection marker in therapeutic strategies for mCRPC patients. However, due to the diversities of patient cohorts and sample characteristics, the varieties of detection methods and different sample types (Tables 1, 2), and the various definitions of AR-V7 positivity and clinical endpoints (Supplementary Tables S1, S2), the clinical utility of AR-V7 as a treatment selection marker in CRPC still needs to be further evaluated.

In this review, a meta-analysis was conducted on 36 studies to explore the prognostic value of AR-V7 in CRPC patients treated with NHT or chemotherapy by the PSA response, OS and PFS. In addition, the conversion of AR-V7 positive proportion after NHT or chemotherapy was further analyzed.

\section{METHODS}

\section{Retrieval Strategy}

This meta-analysis was conducted based on the PRISMA statement and registered at PROSPERO with registration number CRD42020161618 (45). A literature search was performed on PubMed, the Web of Science, and Embase from inception to February 2020. The main search strategy was as follows: (prostate cancer OR prostate tumor OR prostate neoplasm OR prostate carcinoma) AND (AR-V7 OR AR3 OR androgen receptor splicing variant $7 \mathrm{OR}$ androgen receptor 3 ). Bibliographic references of selected articles were examined to identify additional articles that met the selection criteria. Two reviewers independently verified the studies for eligibility and any discrepancies were resolved by a third reviewer.

\section{Selection Criteria}

Retrieved titles and abstracts were screened to eliminate duplicates, and full texts in the selected articles were reviewed and matched against the inclusion criteria. Articles eligible for inclusion met the following criteria: 1) The study reported on CRPC and AR-V7; 2) The results were expressed as a positive rate in percent of AR-V7 before and after treatment in CRPC. Other included results were the PSA response rate, PFS, or OS after NHT or chemotherapy; 3 ) The results were reported from clinical trials including RCTs and nonrandomized studies. Studies were excluded if: 1) treatment was neither novel hormonal therapy nor chemotherapy, or not clearly mentioned; 2) studies reported only the AR-V7-positive proportion before or after treatment in CRPC patients; 3) studies lacked results of therapy response rate, PFS, or OS; 4) studies involved non-human subjects; 5) studies were published in languages other than English except when a translation was provided; 6) case reports, comments, editorials, letters, or reviews. Notably, when more than one publication was retrieved from the same trial, the most recent or most complete report was used.

\section{Data Collection and Study Quality}

1) The following baseline patient characteristics were collected for each eligible trial: age, tumor stage, Gleason score, baseline PSA and alkaline phosphatase, and the median time from diagnosis to sampling. 2) The description of the study design included the author's name, year, country, study design, type of therapy, description of interventions, and outcome measures. 3) The number of patients assigned to different treatment regimens and follow-up duration. 4) The AR-V7-positive percentage before and after treatment in CRPC samples and the methods of detection. 5) The number and proportion of patients with therapy response to NHT or chemotherapy and its detailed definition. 6) The prognosis outcome data were the hazard ratios (HRs) with 95\% confidence intervals (CIs) and Pvalues of PFS, detailed in the subgroup analysis of NHT or chemotherapy. 7) For OS, HRs with 95\% CIs and P-value, detailed in the subgroup analysis of NHT or chemotherapy.

\section{Statistical Methods}

Summary statistics were generated using Review Manager Software (RevMan v.5.3; The Nordic Cochrane Center, 
TABLE 1 | Characteristics of studies included in the change of AR-V7-positive proportion after treatment meta-analysis.

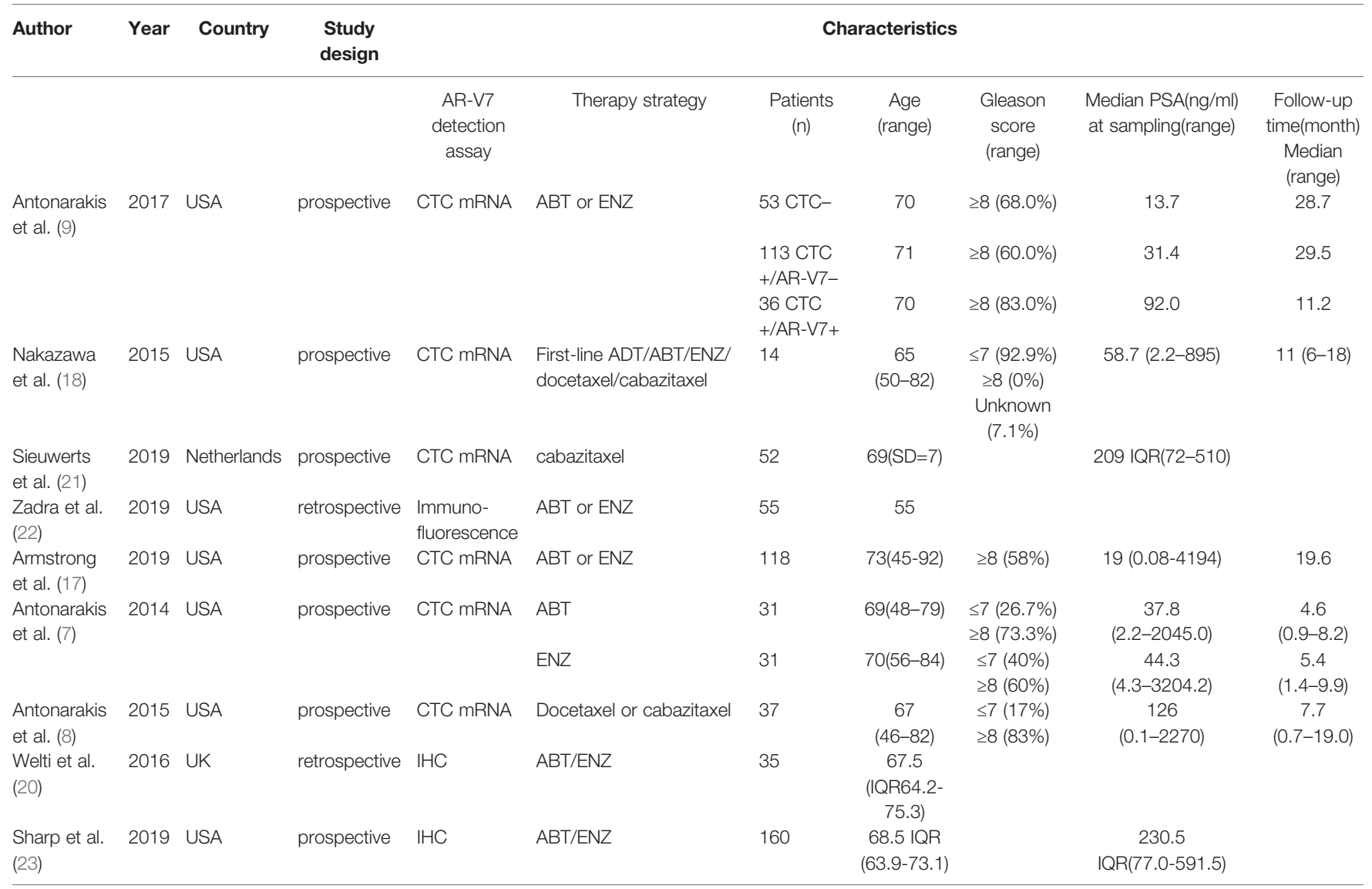

PCa, prostate cancer; CRPC, castration resistance prostate cancer; PSA, prostate specific antigen; IQR, inter quartile range; ABT, Abiraterone; ENZ, Enzalutamide; ADT, androgen deprive therapy; IHC, Immunohistochemistry; CTC, circulating tumor cell.

Copenhagen, Denmark). All patients were included and analyzed on an intention-to-treat basis. The AR-V7-positivity conversion after treatment and the association between AR-V7 expression and the response rate of NHT or chemotherapy were assessed. The primary endpoints of the meta-analysis were OS and PFS. Patients were grouped by AR-V7 status and compared in terms of the PSA response rates, PFS, and OS after NHT or chemotherapy in CRPC. The summary measure for OS and PFS was HR (95\% CI). Statistical differences among studies were evaluated using the chi-square test and the $\mathrm{I}^{2}$ statistic. Odds ratio (OR) and hazard ratio (HR) estimates were weighted by the Mantel-Haenszel method. The pooled effect was calculated using either the fixed effects model $\left(\mathrm{I}^{2}<50 \%\right)$ or the random-effects model $\left(\mathrm{I}^{2} \geq 50 \%\right)$ based on heterogeneity level. All statistical tests were bilateral with a significance $p$ value of $5 \%$.

\section{RESULTS}

\section{Study Characteristics and Quality}

Article selection process is shown in Figure 1. A total of 4347 full-text papers were identified from which 4313 were excluded. Notably, 435 studies were duplicate, 3,381 were not relevant to the research question, 465 were conference abstracts, reviews, letters, and editorials whose quality could not be assessed, and 30 studies did not contain relevant results. No study was identified from reference lists. Eventually, 36 trials were included in the meta-analysis.

\section{Patient Characteristics}

Nine trials including 897 patients were used to compare AR-V7 conversion after treatment. Their clinicopathological features are listed in Table 1. The sample type and AR-V7 detection methods are detailed in Supplementary Table S1. A total of 1,643 patients from 24 studies were enrolled for therapy response comparison; 17 studies including 1,499 patients were used for the comparison of PFS; 1,726 patients from 17 studies were enrolled for OS comparison. Detailed features of these studies are highlighted in Table 2. The definitions of PSA response, PFS, and OS were inconsistent across studies and was displayed in Supplementary Table S2.

\section{AR-V7 Positivity Conversion After Treatment}

A total of 897 patients from nine trials were enrolled. Of note, 154 out of 433 patients were AR-V7 positive before treatment, 
TABLE 2 | Characteristics of studies and patients included in the PSA response, PFS and OS meta-analysis.

\begin{tabular}{|c|c|c|c|c|c|c|c|c|c|c|c|c|}
\hline \multirow[t]{2}{*}{ Study } & \multirow[t]{2}{*}{ Year } & \multirow[t]{2}{*}{ Country } & \multirow{2}{*}{$\begin{array}{l}\text { Study } \\
\text { design }\end{array}$} & \multirow{2}{*}{$\begin{array}{l}\text { AR-V7 detection } \\
\text { assay }\end{array}$} & \multicolumn{8}{|c|}{ Patients characteristics } \\
\hline & & & & & Treatment & $\begin{array}{l}\text { Patients } \\
\text { (n) }\end{array}$ & $\begin{array}{c}\text { Age } \\
\text { (range) }\end{array}$ & $\begin{array}{c}\text { Gleason } \\
\text { score } \\
(\%)\end{array}$ & $\begin{array}{c}\text { Tumor stage } \\
\text { at diagnosis } \\
(\%)\end{array}$ & $\begin{array}{l}\text { Baseline PSA } \\
\text { (ng/ml) median } \\
\text { (range) }\end{array}$ & $\begin{array}{l}\text { Baseline alkaline } \\
\text { phosphatase (U/L) }\end{array}$ & $\begin{array}{c}\text { Follow-up time } \\
\text { (month)Median } \\
\text { (range) }\end{array}$ \\
\hline \multirow[t]{2}{*}{$\begin{array}{l}\text { Antonarakis } \\
\text { et al. (7) }\end{array}$} & 2014 & USA & prospective & CTC mRNA & Abiraterone & 31 & $\begin{array}{c}69 \\
(48-79)\end{array}$ & $\begin{array}{c}\leq 7 \\
(26.7 \%) \\
\geq 8 \\
(73.3 \%)\end{array}$ & $\begin{array}{l}\text { T1/T2 (26.7\%) } \\
\text { T3/T4 (61.3\%) }\end{array}$ & $\begin{array}{c}37.8 \\
(2.2-2045.0)\end{array}$ & $\begin{array}{c}118 \\
(59-1348)\end{array}$ & $\begin{array}{c}4.6 \\
(0.9-8.2)\end{array}$ \\
\hline & & & & & Enzalutamide & 31 & $\begin{array}{c}70 \\
(56-84)\end{array}$ & $\begin{array}{l}\leq 7(40 \%) \\
\geq 8(60 \%)\end{array}$ & $\begin{array}{l}\text { T1/T2 (54.8\%) } \\
\text { T3/T4 (45.2\%) }\end{array}$ & $\begin{array}{c}44.3 \\
(4.3-3204.2)\end{array}$ & $\begin{array}{c}108 \\
(58-872)\end{array}$ & $\begin{array}{c}5.4 \\
(1.4-9.9)\end{array}$ \\
\hline $\begin{array}{l}\text { Antonarakis } \\
\text { et al. (8) }\end{array}$ & 2015 & USA & prospective & CTC mRNA & Docetaxel or cabazitaxel & 37 & $\begin{array}{c}67 \\
(46-82)\end{array}$ & $\begin{array}{l}\leq 7(17 \%) \\
\geq 8(83 \%)\end{array}$ & $\begin{array}{l}\text { T1/T2 (38.0\%) } \\
\text { T3/T4 (62.0\%) }\end{array}$ & $\begin{array}{c}126 \\
(0.1-2270)\end{array}$ & $\begin{array}{c}161 \\
(53-1243)\end{array}$ & $\begin{array}{c}7.7 \\
(0.7-19.0)\end{array}$ \\
\hline \multirow[t]{2}{*}{$\begin{array}{l}\text { Scher et al. } \\
\text { (24) }\end{array}$} & 2016 & USA & cohort study & $\begin{array}{l}\text { CTC } \\
\text { Immunofluorescent }\end{array}$ & $\mathrm{NHT}$ & 130 & $\begin{array}{c}68.5 \\
(45-87)\end{array}$ & - & - & $\begin{array}{c}28.0 \\
(0.1-2454.5)\end{array}$ & $\begin{array}{c}208 \\
(123-1293)\end{array}$ & - \\
\hline & & & & Staining & Taxane Therapy & 63 & $\begin{array}{c}68 \\
(48-91)\end{array}$ & - & - & $\begin{array}{c}99.5 \\
(0.1-3728.2)\end{array}$ & $\begin{array}{c}251.5 \\
(141-1004)\end{array}$ & - \\
\hline $\begin{array}{l}\text { Steinestel } \\
\text { et al. (25) }\end{array}$ & 2015 & Germany & prospective & CTC mRNA & $\begin{array}{l}\text { Enzalutamide or } \\
\text { Abiraterone }\end{array}$ & 24 & $\begin{array}{c}75 \\
(53-87)\end{array}$ & $\begin{array}{c}\leq 7 \\
(41.3 \%) \\
\geq 8 \\
(58.7 \%)\end{array}$ & - & $\begin{array}{c}96.5 \\
(0.1-4282)\end{array}$ & - & - \\
\hline $\begin{array}{l}\text { Todenhofer } \\
\text { et al. (26) }\end{array}$ & 2016 & Canada & prospective & CTC mRNA & Abiraterone & 37 & $\begin{array}{c}70 \\
(53-87)\end{array}$ & - & - & - & $\begin{array}{c}116 \\
(45-1869)\end{array}$ & - \\
\hline $\begin{array}{l}\text { Welti et al. } \\
(20)\end{array}$ & 2016 & UK & retrospective & $\mathrm{IHC}$ & NHT or chemotherapy & 35 & $\begin{array}{c}67.5 \\
\text { (IQR64.2- } \\
75.3)\end{array}$ & - & - & - & $\begin{array}{c}142.0 \\
(69.5-448.5)\end{array}$ & - \\
\hline $\begin{array}{l}\text { Onstenk } \\
\text { et al. (13) }\end{array}$ & 2015 & Netherlands & prospective & CTC mRNA & Cabazitaxel & 29 & $\begin{array}{c}70 \\
(\mathrm{SD} \pm 7)\end{array}$ & - & - & $\begin{array}{c}321 \\
(\text { IQR76-649) }\end{array}$ & $\begin{array}{c}163 \\
(106-375)\end{array}$ & $\begin{array}{c}7 \\
(2-27)\end{array}$ \\
\hline \multirow[t]{2}{*}{$\begin{array}{l}\text { Qu F et al. } \\
\text { (27) }\end{array}$} & 2016 & USA & retrospective & CTC mRNA & Abiraterone & 81 & $\begin{array}{c}68.3 \\
(46-89)\end{array}$ & $\begin{array}{c}\leq 7 \\
(41.9 \%) \\
\geq 8 \\
(49.4 \%) \\
\text { Unknown } \\
(8.6 \%)\end{array}$ & - & $\begin{array}{c}16.4 \\
(0.1-972.1)\end{array}$ & - & $\begin{array}{c}29.7 \\
(3.6-47.5)\end{array}$ \\
\hline & & & & & Enzalutamide & 51 & $\begin{array}{l}69.0 \\
(5-88)\end{array}$ & $\begin{array}{c}\leq 7 \\
(47.1 \%) \\
\geq 8 \\
(41.2 \%) \\
\text { Unknown } \\
(11.8 \%)\end{array}$ & - & $\begin{array}{c}45.5 \\
(0.3-1148.4)\end{array}$ & - & $\begin{array}{c}23.9 \\
(0.9-48.3)\end{array}$ \\
\hline \multirow[t]{3}{*}{$\begin{array}{l}\text { Antonaraki } \\
\text { et al. (9) }\end{array}$} & 2017 & USA & prospective & CTC mRNA & $\begin{array}{l}\text { Enzalutamide or } \\
\text { Abiraterone }\end{array}$ & 53 CTC- & 70 & $\begin{array}{c}\geq 8 \\
(68.0 \%)\end{array}$ & - & 13.7 & 80 & 28.7 \\
\hline & & & & & & $\begin{array}{c}113 \text { CTC } \\
+/ \text { AR- } \\
\text { V7- }\end{array}$ & 71 & $\begin{array}{c}\geq 8 \\
(60.0 \%)\end{array}$ & - & 31.4 & 96 & 29.5 \\
\hline & & & & & & $\begin{array}{l}36 \text { CTC } \\
+/ \text { AR-V7+ }\end{array}$ & 70 & $\begin{array}{c}\geq 8 \\
(83.0 \%)\end{array}$ & - & 92.0 & 120 & 11.2 \\
\hline
\end{tabular}




\begin{tabular}{|c|c|c|c|c|c|c|c|c|c|c|c|c|}
\hline \multirow[t]{2}{*}{ Study } & \multirow[t]{2}{*}{ Year } & \multirow[t]{2}{*}{ Country } & \multirow{2}{*}{$\begin{array}{l}\text { Study } \\
\text { design }\end{array}$} & \multirow{2}{*}{$\begin{array}{l}\text { AR-V7 detection } \\
\text { assay }\end{array}$} & \multicolumn{8}{|c|}{ Patients characteristics } \\
\hline & & & & & Treatment & $\begin{array}{l}\text { Patients } \\
\text { (n) }\end{array}$ & $\begin{array}{c}\text { Age } \\
\text { (range) }\end{array}$ & $\begin{array}{l}\text { Gleason } \\
\text { score } \\
(\%)\end{array}$ & $\begin{array}{c}\text { Tumor stage } \\
\text { at diagnosis } \\
(\%)\end{array}$ & $\begin{array}{l}\text { Baseline PSA } \\
\text { (ng/ml) median } \\
\text { (range) }\end{array}$ & $\begin{array}{l}\text { Baseline alkaline } \\
\text { phosphatase (U/L) }\end{array}$ & $\begin{array}{c}\text { Follow-up time } \\
\text { (month)Median } \\
\text { (range) }\end{array}$ \\
\hline $\begin{array}{l}\text { Nakazawa } \\
\text { et al. (18) }\end{array}$ & 2015 & USA & prospective & CTC mRNA & NHT or chemotherapy & 14 & $\begin{array}{c}65 \\
(50-82)\end{array}$ & $\begin{array}{c}\leq 7 \\
(92.9 \%) \\
\geq 8(0 \%) \\
\text { Unknown } \\
(7.1 \%)\end{array}$ & & $58.7(2.2-895)$ & $\begin{array}{c}127 \\
(52-838)\end{array}$ & $\begin{array}{c}11 \\
(6-18)\end{array}$ \\
\hline $\begin{array}{l}\text { Del Re et al. } \\
\text { (28) }\end{array}$ & 2017 & Italy & prospective & $\begin{array}{l}\text { Plasma exosomal } \\
\text { RNA }\end{array}$ & $\begin{array}{l}\text { Enzalutamide or } \\
\text { Abiraterone }\end{array}$ & 36 & $\begin{array}{c}66 \\
(51-81)\end{array}$ & $\begin{array}{c}\leq 7(44 \%) \\
\geq 8(53 \%) \\
\text { Unknown } \\
(3 \%)\end{array}$ & $\begin{array}{l}\text { T1/T2 (8.0\%) } \\
\text { T3/T4 (36.0\%) }\end{array}$ & $\begin{array}{c}26.3 \\
(0.63-4581)\end{array}$ & $\begin{array}{c}180 \\
(49-917)\end{array}$ & $\begin{array}{c}9 \\
(2.0-31.0)\end{array}$ \\
\hline $\begin{array}{l}\text { Zhu et al. } \\
(24)\end{array}$ & 2018 & USA & retrospective & $\mathrm{RISH}$ & $\begin{array}{l}\text { Enzalutamide or } \\
\text { Abiraterone }\end{array}$ & 28 & $\begin{array}{c}64 \\
(52-86)\end{array}$ & $\begin{array}{c}\leq 7 \\
(21.4 \%) \\
\geq 8 \\
(78.6 \%)\end{array}$ & & $\begin{array}{c}59.6 \\
(0.7-6746.8)\end{array}$ & & \\
\hline & & UK & retrospective & $\mathrm{RISH}$ & $\begin{array}{l}\text { Enzalutamide or } \\
\text { Abiraterone }\end{array}$ & 16 & $\begin{array}{l}72.3 \\
(48.8- \\
79.4)\end{array}$ & $\begin{array}{c}\leq 7(25 \%) \\
\geq 8(50 \%) \\
\text { Unknown } \\
(25 \%)\end{array}$ & & $\begin{array}{c}177.0 \\
(2.6-4098.0)\end{array}$ & & \\
\hline $\begin{array}{l}\text { To et al. } \\
\text { (14) }\end{array}$ & 2018 & Australia & prospective & $\begin{array}{l}\text { whole blood } \\
\text { mRNA }\end{array}$ & $\begin{array}{l}\text { Enzalutamide or } \\
\text { Abiraterone }\end{array}$ & $\begin{array}{l}9 \text { AR-V } \\
\text { positive }\end{array}$ & $\begin{array}{l}77(46- \\
89)\end{array}$ & $\begin{array}{c}\leq 7 \\
(44.4 \%) \\
\geq 8 \\
(55.6 \%)\end{array}$ & & $\begin{array}{c}49.2 \\
(3-703)\end{array}$ & & \\
\hline & & & & & & $\begin{array}{l}28 \text { AR-V } \\
\text { negative }\end{array}$ & $\begin{array}{c}75.5(52- \\
89)\end{array}$ & $\begin{array}{c}\leq 7 \\
(14.3 \%) \\
\geq 8 \\
(46.4 \%) \\
\text { Unknown } \\
(39.3 \%)\end{array}$ & & $42.5(0.8-588)$ & & \\
\hline $\begin{array}{l}\text { Seitz et al. } \\
(29)\end{array}$ & 2017 & Germany & prospective & $\begin{array}{l}\text { whole blood } \\
\text { mRNA }\end{array}$ & $\begin{array}{l}\text { Enzalutamide or } \\
\text { Abiraterone }\end{array}$ & 85 & $\begin{array}{l}71(66- \\
74)\end{array}$ & & & $\begin{array}{c}211 \\
\text { (IQR29-768) }\end{array}$ & & 7.6 (IQR4.7-12.7) \\
\hline $\begin{array}{l}\text { De Laere } \\
\text { et al. (30) }\end{array}$ & 2017 & Belgium & retrospective & CTC mRNA & $\begin{array}{l}\text { Enzalutamide or } \\
\text { Abiraterone }\end{array}$ & 17 & & & & & & \\
\hline $\begin{array}{l}\text { Scher et al. } \\
\text { (31) }\end{array}$ & 2017 & USA & prospective & CTC mRNA & $\begin{array}{l}\text { Enzalutamide or } \\
\text { Abiraterone }\end{array}$ & 161 & $\begin{array}{l}68(45- \\
91)\end{array}$ & $\begin{array}{c}8 \text { (range } \\
5-10)\end{array}$ & & $37.7(0.1-3728.2)$ & $111(25-2170)$ & $\begin{array}{c}11 \\
(1-30)\end{array}$ \\
\hline $\begin{array}{l}\text { Takeuchi } \\
\text { et al. (13) }\end{array}$ & 2016 & Japan & cohort study & $\begin{array}{l}\text { whole blood } \\
\text { mRNA }\end{array}$ & $\begin{array}{l}\text { Enzalutamide or } \\
\text { Abiraterone }\end{array}$ & 43 & $\begin{array}{c}73 \\
(59-88)\end{array}$ & $\begin{array}{c}\leq 7 \\
(20.9 \%) \\
\geq 8 \\
(72.1 \%) \\
\text { Unknown } \\
(7.0 \%)\end{array}$ & & $130(5.3-9529)$ & & \\
\hline $\begin{array}{l}\text { Okegawa } \\
\text { et al. (32) }\end{array}$ & 2018 & Japan & retrospective & CTC mRNA & $\begin{array}{l}\text { Enzalutamide or } \\
\text { Abiraterone }\end{array}$ & 49 CTC- & 69 & $\begin{array}{c}\geq 8 \\
(81.6 \%)\end{array}$ & & 75.7 & 317 & 20.7 (3.0-37.0) \\
\hline & & & & & & $\begin{array}{l}23 \mathrm{CTC}+ \\
\text { AR-V7- }\end{array}$ & 71 & $\begin{array}{c}\geq 8 \\
(91.3 \%)\end{array}$ & & 71.5 & 323 & \\
\hline & & & & & & & 72 & & & 79.1 & 378 & \\
\hline
\end{tabular}




\begin{tabular}{|c|c|c|c|c|c|c|c|c|c|c|c|c|}
\hline \multirow[t]{2}{*}{ Study } & \multirow[t]{2}{*}{ Year } & \multirow[t]{2}{*}{ Country } & \multirow{2}{*}{$\begin{array}{l}\text { Study } \\
\text { design }\end{array}$} & \multirow{2}{*}{$\begin{array}{l}\text { AR-V7 detection } \\
\text { assay }\end{array}$} & \multicolumn{8}{|c|}{ Patients characteristics } \\
\hline & & & & & Treatment & $\begin{array}{l}\text { Patients } \\
\text { (n) }\end{array}$ & $\begin{array}{c}\text { Age } \\
\text { (range) }\end{array}$ & $\begin{array}{l}\text { Gleason } \\
\text { score } \\
(\%)\end{array}$ & $\begin{array}{c}\text { Tumor stage } \\
\text { at diagnosis } \\
(\%)\end{array}$ & $\begin{array}{c}\text { Baseline PSA } \\
\text { (ng/ml) median } \\
\text { (range) }\end{array}$ & $\begin{array}{l}\text { Baseline alkaline } \\
\text { phosphatase (U/L) }\end{array}$ & $\begin{array}{c}\text { Follow-up time } \\
\text { (month)Median } \\
\text { (range) }\end{array}$ \\
\hline $\begin{array}{l}\text { Kohli et al. } \\
\text { (33) }\end{array}$ & 2017 & USA & prospective & $\begin{array}{l}\text { CTC mRNA and } \\
\text { biopsies mRNA }\end{array}$ & Abiraterone & $\begin{array}{c}26 \text { CTC+ } \\
\text { AR-V7+ } \\
78\end{array}$ & & $\begin{array}{c}\geq 8 \\
(96.2 \%)\end{array}$ & & & & \\
\hline $\begin{array}{l}\text { Tommasi } \\
\text { et al. (34) }\end{array}$ & 2018 & Italy & prospective & CTC mRNA & $\begin{array}{l}\text { Enzalutamide or } \\
\text { Abiraterone, LHRH, } \\
\text { Chemotherapy }\end{array}$ & 44 & $\begin{array}{l}71.5(55- \\
87)\end{array}$ & $\begin{array}{c}\leq 7(6.8 \%) \\
\geq 8 \\
(81.8 \%) \\
\text { Unknown } \\
(11.4 \%)\end{array}$ & $\begin{array}{c}\text { T1/T2 (15.9\%) } \\
\text { T3/T4 (79.5\%) } \\
\text { Unknown } \\
(4.5 \%)\end{array}$ & 39 (0.005-2896) & - & 20.5 \\
\hline $\begin{array}{l}\text { Armstrong } \\
\text { et al. (17) }\end{array}$ & 2019 & USA & prospective & CTC mRNA & $\begin{array}{l}\text { Enzalutamide or } \\
\text { Abiraterone }\end{array}$ & 118 & $\begin{array}{l}73(45- \\
92)\end{array}$ & $\geq 8(58 \%)$ & M1(32\%) & $\begin{array}{c}19 \\
(0.08-4194)\end{array}$ & $40 \%$ Elevated & 19.6 \\
\hline $\begin{array}{l}\text { Sharp et al. } \\
\text { (19) }\end{array}$ & 2019 & USA & prospective & $\mathrm{IHC}$ & $\begin{array}{l}\text { Enzalutamide or } \\
\text { Abiraterone, } \\
\text { Chemotherapy }\end{array}$ & 160 & $\begin{array}{c}68.5 \mathrm{IQR} \\
(63.9- \\
73.1)\end{array}$ & & $\begin{array}{l}\mathrm{M} 1 \mathrm{~b}(67 \%) \\
\mathrm{M} 1 \mathrm{c}(21 \%)\end{array}$ & $\begin{array}{c}230.5 \text { IQR(77.0- } \\
591.5)\end{array}$ & $\begin{array}{c}\text { 127.0 IQR(72.3- } \\
332.5)\end{array}$ & \\
\hline $\begin{array}{l}\text { Sieuwerts } \\
\text { et al. (21) }\end{array}$ & 2019 & Netherlands & prospective & CTC mRNA & cabazitaxel & 52 & $69(\mathrm{SD}=7)$ & & & 209 IQR(72-510) & 174 IQR(98-339) & \\
\hline $\begin{array}{l}\text { Cattrini } \\
\text { et al. (35) }\end{array}$ & 2019 & Italy & prospective & CTC mRNA & $\begin{array}{l}\text { Enzalutamide, } \\
\text { Abiraterone or Docetaxel }\end{array}$ & 39 & $\begin{array}{c}72 \\
(56-84)\end{array}$ & & $\begin{array}{l}\operatorname{M1b}(79.5 \%) \\
\operatorname{M1c}(17.9 \%)\end{array}$ & $35.2(0.33-4688)$ & & \\
\hline $\begin{array}{l}\text { Chung et al. } \\
\text { (36) }\end{array}$ & 2019 & USA & prospective & CTC mRNA & $\begin{array}{l}\text { Enzalutamide or } \\
\text { Abiraterone }\end{array}$ & 37 & $\begin{array}{c}72 \\
(67-79)\end{array}$ & $\begin{array}{c}\leq 7 \\
(43.2 \%) \\
8(8.1 \%) \\
\geq 9(46 \%) \\
\text { Unknown } \\
(2.7 \%)\end{array}$ & $\begin{array}{l}\text { N1(64.9\%) } \\
\text { M1b(89.2\%) } \\
\text { M1c(27\%) }\end{array}$ & $\begin{array}{c}20.9 \\
\operatorname{IQR}(11.6-96.8)\end{array}$ & $\begin{array}{c}102.0 \\
\operatorname{IQR}(80.5-170.5)\end{array}$ & $\begin{array}{l}11.43(\mathrm{IQR}: \\
4.73-21.3)\end{array}$ \\
\hline $\begin{array}{l}\text { Del Re et al. } \\
\text { (37) }\end{array}$ & 2019 & Italy & retrospective & $\begin{array}{l}\text { Plasma exosomal } \\
\text { RNA }\end{array}$ & $\begin{array}{l}\text { First time } \\
\text { Enzalutamide or } \\
\text { Abiraterone }\end{array}$ & 46 & & $\begin{array}{c}\leq 7 \\
(43.5 \%) \\
\geq 8 \\
(47.8 \%) \\
\text { Unknown } \\
(8.7 \%)\end{array}$ & $\begin{array}{c}\text { T1/T2 (13\%) } \\
\text { T3/T4 (13\%) } \\
\text { N1(21.8\%) } \\
\text { M1(21.8\%) }\end{array}$ & & & \\
\hline & & & & & $\begin{array}{l}\text { second time } \\
\text { Enzalutamide or } \\
\text { Abiraterone }\end{array}$ & 27 & & $\begin{array}{c}\leq 7(52 \%) \\
\geq 8(38 \%) \\
\text { Unknown } \\
(10 \%)\end{array}$ & $\begin{array}{c}\mathrm{T} 1 / \mathrm{T} 2(11.1 \%) \\
\mathrm{T} 3 / \mathrm{T} 4 \\
(25.9 \%) \mathrm{N} 1 \\
(14.8 \%) \\
\mathrm{M} 1(40.8 \%)\end{array}$ & & & \\
\hline $\begin{array}{l}\text { El-Heliebi } \\
\text { et al. (38) }\end{array}$ & 2018 & $\begin{array}{l}\text { Austria } \\
\text { Germany } \\
\text { Netherlands } \\
\text { Sweden }\end{array}$ & prospective & CTC FISH & $\begin{array}{l}\text { Enzalutamide, } \\
\text { Abiraterone or Taxane }\end{array}$ & 31 & $\begin{array}{c}70.5 \\
(42-83)\end{array}$ & $\begin{array}{c}\leq 7 \\
(38.7 \%) \\
\geq 8 \\
(51.6 \%)\end{array}$ & $\begin{array}{c}\mathrm{T} 1 / \mathrm{T} 2(22.6 \%) \\
\mathrm{T} 3 / \mathrm{T} 4 \\
(45.2 \%)\end{array}$ & $\begin{array}{c}48.81 \\
(0.8-4623)\end{array}$ & & \\
\hline $\begin{array}{l}\text { Tagawa } \\
\text { et al. (15) }\end{array}$ & 2019 & USA & prospective & CTC mRNA & Docetaxel or Cabazitaxel & 54 & $\begin{array}{c}71(53- \\
84)\end{array}$ & $\begin{array}{c}\leq 6 \\
(13.7 \%) \\
7(25.5 \%) \\
\geq 8 \\
(60.8 \%)\end{array}$ & $\begin{array}{l}\mathrm{N} 1(51.9 \%) \\
\mathrm{M} 1 \mathrm{~b}(90.7 \%) \\
\mathrm{M} 1 \mathrm{c}(40.7 \%)\end{array}$ & $\begin{array}{c}92.1 \\
(2.4-1558)\end{array}$ & $217.8(\mathrm{SD}=260.35)$ & \\
\hline
\end{tabular}




\begin{tabular}{|c|c|c|c|c|c|c|c|c|c|c|c|c|}
\hline \multirow[t]{2}{*}{ Study } & \multirow[t]{2}{*}{ Year } & \multirow[t]{2}{*}{ Country } & \multirow{2}{*}{$\begin{array}{l}\text { Study } \\
\text { design }\end{array}$} & \multirow{2}{*}{$\begin{array}{l}\text { AR-V7 detection } \\
\text { assay }\end{array}$} & \multicolumn{8}{|c|}{ Patients characteristics } \\
\hline & & & & & Treatment & $\begin{array}{l}\text { Patients } \\
\text { (n) }\end{array}$ & $\begin{array}{c}\text { Age } \\
\text { (range) }\end{array}$ & $\begin{array}{l}\text { Gleason } \\
\text { score } \\
(\%)\end{array}$ & $\begin{array}{l}\text { Tumor stage } \\
\text { at diagnosis } \\
(\%)\end{array}$ & $\begin{array}{c}\text { Baseline PSA } \\
\text { (ng/ml) median } \\
\text { (range) }\end{array}$ & $\begin{array}{l}\text { Baseline alkaline } \\
\text { phosphatase (U/L) }\end{array}$ & $\begin{array}{c}\text { Follow-up time } \\
\text { (month)Median } \\
\text { (range) }\end{array}$ \\
\hline $\begin{array}{l}\text { Worroll } \\
\text { et al. (39) }\end{array}$ & 2019 & USA & prospective & $\begin{array}{l}\text { CTC } \\
\text { Immunofluorescence }\end{array}$ & Taxane & 15 & & & & & & \\
\hline $\begin{array}{l}\text { Belderbos } \\
\text { et al. ( } 40)\end{array}$ & 2019 & Netherlands & prospective & CTC mRNA & $\begin{array}{l}\text { Enzalutamide, } \\
\text { Abiraterone or } \\
\text { Cabazitaxel }\end{array}$ & 94 & $\begin{array}{l}69 \mathrm{IQR} \\
(65-75)\end{array}$ & & & 186 IQR (67-356) & & \\
\hline \multirow[t]{3}{*}{$\begin{array}{l}\text { Sharp et al. } \\
\text { (23) }\end{array}$} & 2019 & UK/USA & prospective & $\begin{array}{l}\text { CTC mRNA } \\
/ / \mathrm{HC}\end{array}$ & $\begin{array}{l}\text { Enzalutamide, } \\
\text { Abiraterone or Taxane }\end{array}$ & 95 CTC- & $\begin{array}{c}71.0 \text { IQR } \\
(66.8- \\
75.6)\end{array}$ & & $\begin{array}{l}\mathrm{M} 1 \mathrm{~b}(74.7 \%) \\
\mathrm{M} 1 \mathrm{c}(17.9 \%)\end{array}$ & $\begin{array}{l}110.0 \text { IQR } \\
(29-300.5)\end{array}$ & $\begin{array}{c}83.0 \text { IQR (66.0- } \\
\text { 163.0) }\end{array}$ & \\
\hline & & & & & & $\begin{array}{c}86 \text { CTC+ } \\
\text { ARV7- }\end{array}$ & $\begin{array}{c}\text { 69.6 IQR } \\
(64.9- \\
72.3)\end{array}$ & & $\begin{array}{l}\text { M1b(86.1\%) } \\
\text { M1c(24.4\%) }\end{array}$ & $\begin{array}{l}147.0 \mathrm{IQR} \\
(51.0-345)\end{array}$ & $\begin{array}{c}111.5 \text { IQR } \\
(76.3-200.5)\end{array}$ & \\
\hline & & & & & & $\begin{array}{c}96 \mathrm{CTC}+ \\
\mathrm{ARV}+\end{array}$ & $\begin{array}{c}70.4 \\
(65.3- \\
74.6)\end{array}$ & & $\begin{array}{l}\text { M1b(84.4\%) } \\
\text { M1c(24.0\%) }\end{array}$ & $\begin{array}{c}244.5 \text { IQR } \\
(109.3-746.8)\end{array}$ & $\begin{array}{c}180.0 \text { IQR } \\
(93.8-346.0)\end{array}$ & \\
\hline $\begin{array}{l}\text { Maillet et al. } \\
(41)\end{array}$ & 2019 & France & prospective & CTC mRNA & $\begin{array}{l}\text { Enzalutamide or } \\
\text { Abiraterone }\end{array}$ & 41 & 73 & $\geq 8(56 \%)$ & M1(29\%) & 35 & & $\begin{array}{c}10.5(95 \% \mathrm{Cl} 8.7- \\
13.7)\end{array}$ \\
\hline $\begin{array}{l}\text { Graf et al. } \\
\text { (42) }\end{array}$ & 2019 & USA & prospective & CTC mRNA & $\begin{array}{l}\text { Enzalutamide/ } \\
\text { Abiraterone or Taxane }\end{array}$ & 193 & $\begin{array}{l}69 \mathrm{IQR} \\
(62.5- \\
75)\end{array}$ & & $\begin{array}{l}\operatorname{M1b}(89 \%) \\
\operatorname{M1c}(22 \%)\end{array}$ & $\begin{array}{c}50.4 \mathrm{IQR} \\
(18.2-211.2)\end{array}$ & $\begin{array}{c}111 \text { IQR } \\
(80-199.5)\end{array}$ & \\
\hline $\begin{array}{l}\text { Erb et al. } \\
\text { (43) }\end{array}$ & 2020 & Germany & prospective & CTC $\Vdash \mathrm{HC}$ & $\begin{array}{l}\text { Enzalutamide/ } \\
\text { Abiraterone or Taxane }\end{array}$ & 26 & $74.3 \pm 9$ & & $\begin{array}{l}\mathrm{N} 1(65.4 \%) \\
\mathrm{M} 1 \mathrm{~b}(84.6 \%) \\
\mathrm{M} 1 \mathrm{c}(19.2 \%)\end{array}$ & & & \\
\hline $\begin{array}{l}\text { Kwan et al. } \\
(44)\end{array}$ & 2020 & Australia & prospective & $\begin{array}{l}\text { whole blood } \\
\text { mRNA }\end{array}$ & $\begin{array}{l}\text { Enzalutamide/ } \\
\text { Abiraterone or Taxane }\end{array}$ & 115 & $\begin{array}{c}72(46- \\
91)\end{array}$ & $\begin{array}{l}\leq 7(24 \%) \\
\geq 8(50 \%)\end{array}$ & M1(53\%) & 42 (0.51-2719) & $131(45-5918)$ & $\begin{array}{c}15.5 \\
(1.4-29)\end{array}$ \\
\hline
\end{tabular}

IQR, inter quartile range; $S D$, standard deviation; $A R-V 7$, androgen receptor splice variant 7; CTC, circulating tumor cell; $P S A$, prostate specific antigen; $A D T$, androgen deprivation therapy; $N H T$, novel hormonal therapy; $L H R H$, Iuteinizing hormone releasing hormone. 


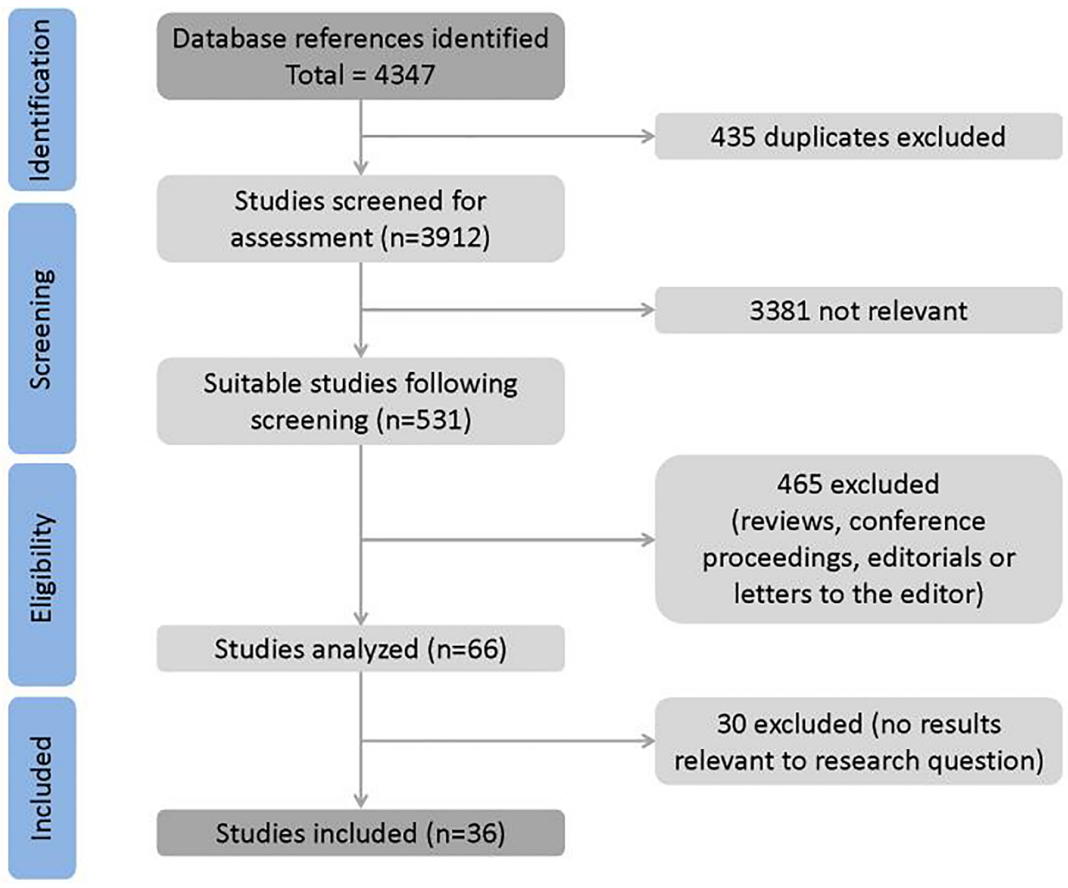

FIGURE 1 | Study selection process.

however, the proportion converted to 243 out of 464 after treatment. Subgroup analysis of different treatment strategies was performed (Figure 2). A significant study heterogeneity was detected $\left(\mathrm{I}^{2}=71 \%, \mathrm{P}<0.001\right)$, and thus a random-effects model was used. The proportion of AR-V7 positivity significantly increased after Abiraterone or Enzalutamide treatment (OR 2.56, 95\% CI 1.51-4.32, $\mathrm{P}<0.001)$ whereas, it significantly declined after chemotherapy (OR $0.51,95 \%$ CI $0.28-$ 0.93, $\mathrm{P}=0.003$ ).

\section{The Relationship Between AR-V7 Expression and PSA Response in CRPC Patients}

In total, 61 out of $347 \mathrm{AR}-\mathrm{V} 7$-positive men showed good response to NHT whereas, 544 out of $985 \mathrm{AR}-\mathrm{V7}$-negative CRPC showed

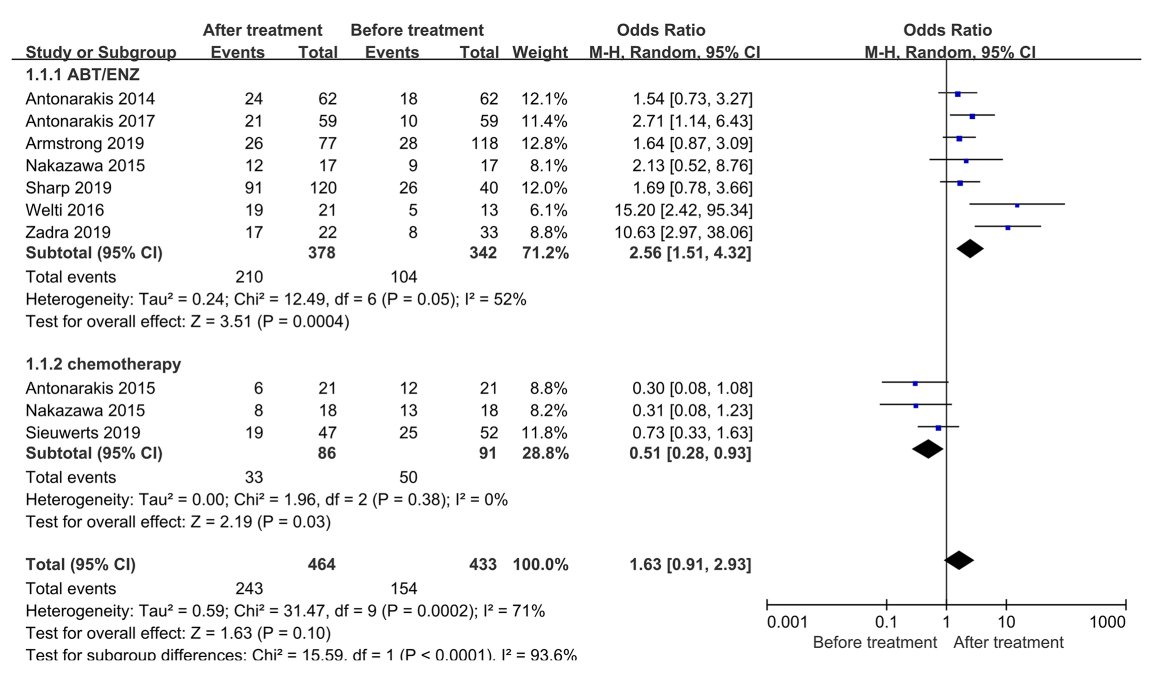

FIGURE 2 | Forest plots of AR-V7-positive proportion conversion after treatment from nine studies. AR-V7-positive proportion before and after treatment were calculated using random-effect models. The bars indicate 95\% Cls. AR-V7, androgen receptor splicing variant 7; ABT, Abiraterone; ENZ, Enzalutamide; Cl, confidence interval; OR, odds ratio. 
good response to PSA (Figure 3A). AR-V7-positive patients had significantly lower PSA response after NHT treatment compared to AR-V7-negative (OR 0.13, 95\% CI 0.09-0.13, $\mathrm{P}<0.001$ ). However, no significant heterogeneity was observed among the studies in this effect $\left(\mathrm{I}^{2}=0.0 \%, \mathrm{P}=0.80\right)$. In subgroup analysis, the OR of a PSA response in AR-V7-positive CRPC was 0.07 (95\% CI 0.02-0.31, $\mathrm{P}<0.001)$ for Abiraterone-treated group, 0.06 (95\% CI
0.01-0.29, $\mathrm{P}<0.001)$ for Enzalutamide group, and 0.14 (95\% CI $0.10-0.21, \mathrm{P}<0.001)$ for Abiraterone or Enzalutamide subgroup. Similarly, 65 out of 165 AR-V7 positive patients showed therapeutic response to chemotherapy whereas, in AR-V7 negative patients, the proportion was $48.5 \%$ (83 of 171), (Figure 3B). Moreover, the PSA response rate was decreased in AR-V7 positive patients (OR $0.63,95 \%$ CI $0.40-1.01, \mathrm{P}=0.06$ ). A fixed-

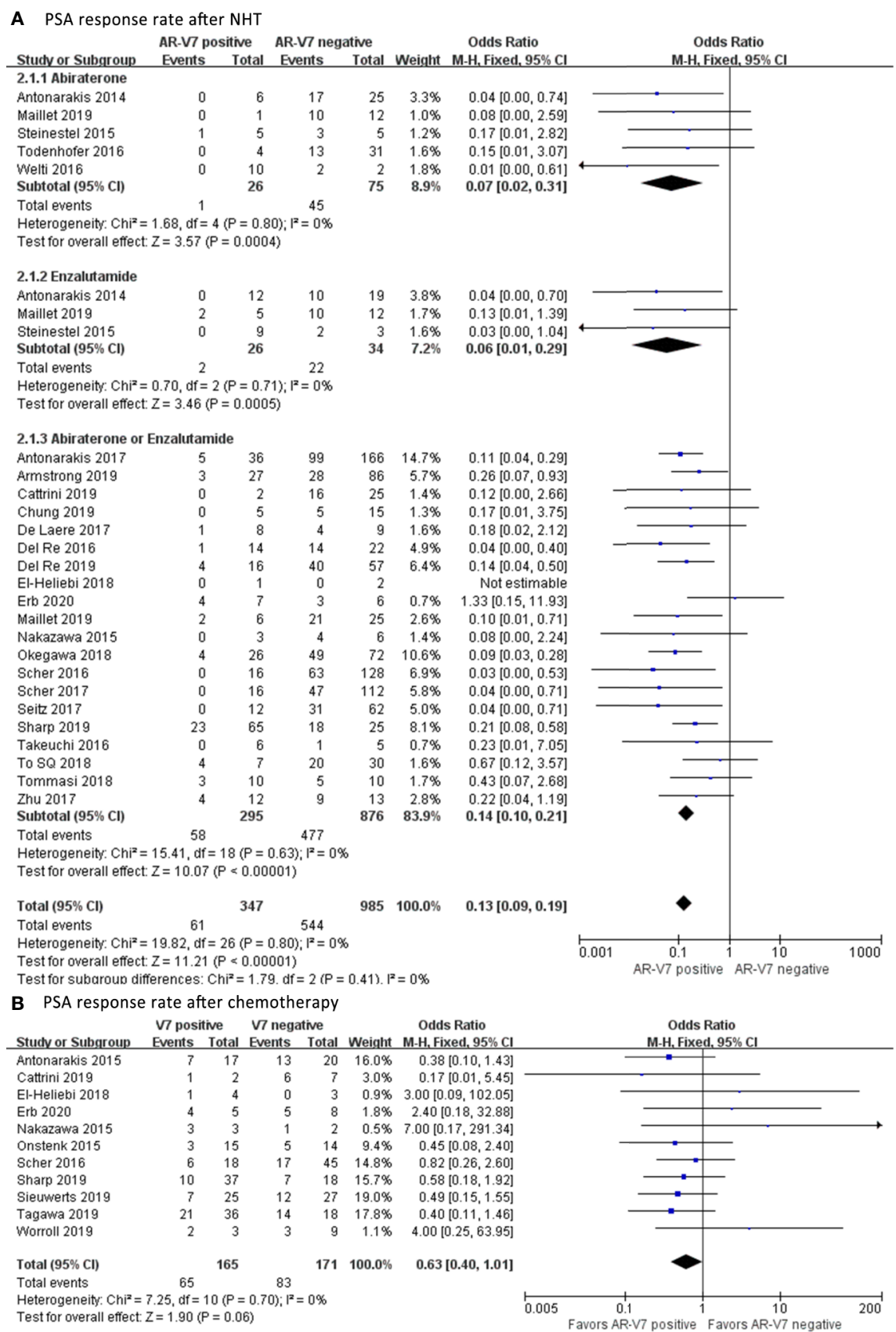

FIGURE 3 | Forest plots of PSA response rate after NHT and chemotherapy. The PSA response rate in AR-V7-positive and AR-V7-negative patients after NHT and chemotherapy was calculated using fixed-effect models. (A) PSA response rate after NHT. (B) PSA response rate after chemotherapy. The bars indicate 95\% Cls. NHT, novel hormonal therapy; ABT, Abiraterone; ENZ, Enzalutamide; PSA, prostate specific antigen; AR-V7, androgen receptor splicing variant 7; Cl, confidence interval. 
effects model was applied since there was no discrepancy between studies $\left(\mathrm{I}^{2}=0.0 \%, \mathrm{P}=0.70\right)$.

\section{The Effect of AR-V7 Expression on Progression-Free Survival in CRPC Patients}

AR-V7 negative patients benefited more from NHT in terms of PFS compared with the positive patients (HR 3.56, 95\% CI 2.535.01, $\mathrm{P}<0.001$ ), (Figure 4A). Notable study discrepancy was tested $\left(\mathrm{I}^{2}=53 \%, \mathrm{P}=0.007\right)$ where a random effect model was applied for this analysis. Further subgroup analysis revealed that AR-V7 positive patients had worse PFS following Abiraterone treatment (HR 4.07, 95\% CI 1.26-13.16, P=0.02), Enzalutamide treatment (HR 3.66, 95\% CI 1.03-12.99, $\mathrm{P}=0.04$ ), and Abiraterone/Enzalutamide treatment (HR 3.49, 95\% CI 2.554.77, $\mathrm{P}<0.001)$. However, patient with AR-V7-negative status tended to have a better PFS although not significantly (HR 1.35, 95\% CI 0.97-1.87, P=0.07), (Figure 4B). There was no heterogeneity between the studies $\left(\mathrm{I}^{2}=45 \%, \mathrm{P}=0.12\right)$, therefore, the fixed-effects model was applied.

\section{The Effect of AR-V7 Expression on Overall Survival of CRPC Patients}

Among all patients who received NHT, AR-V7 negative patients had the best OS (HR 4.47, 95\% CI 3.03-6.59, P<0.001), (Figure 5A). A significant study difference was detected $\left(\mathrm{I}^{2}=49 \%\right.$, $\mathrm{p}=0.03$ ), thus a random effect model was applied. Further subgroup analyses indicated that AR-V7 positive patients had a worse OS after Abiraterone treatment (HR 4.06, 95\% CI 1.2113.64, $\mathrm{P}=0.02$ ), Enzalutamide (HR 3.35, 95\% CI 1.06-10.61, $\mathrm{P}=0.04$ ), and Abiraterone/Enzalutamide treatment (HR 5.25, 95\% CI 3.49-7.89, $\mathrm{P}<0.001)$. For patients who received chemotherapy, AR-V7 positive patients had a worse OS than negative patients (Figure 5B, HR 1.98, 95\% CI 1.48-2.66, $\mathrm{P}<0.001)$. A similar result was observed for non-standardized treatment (Figure 5C, HR 3.26, 95\% CI 1.71-6.22, $\mathrm{P}<0.001$ ).

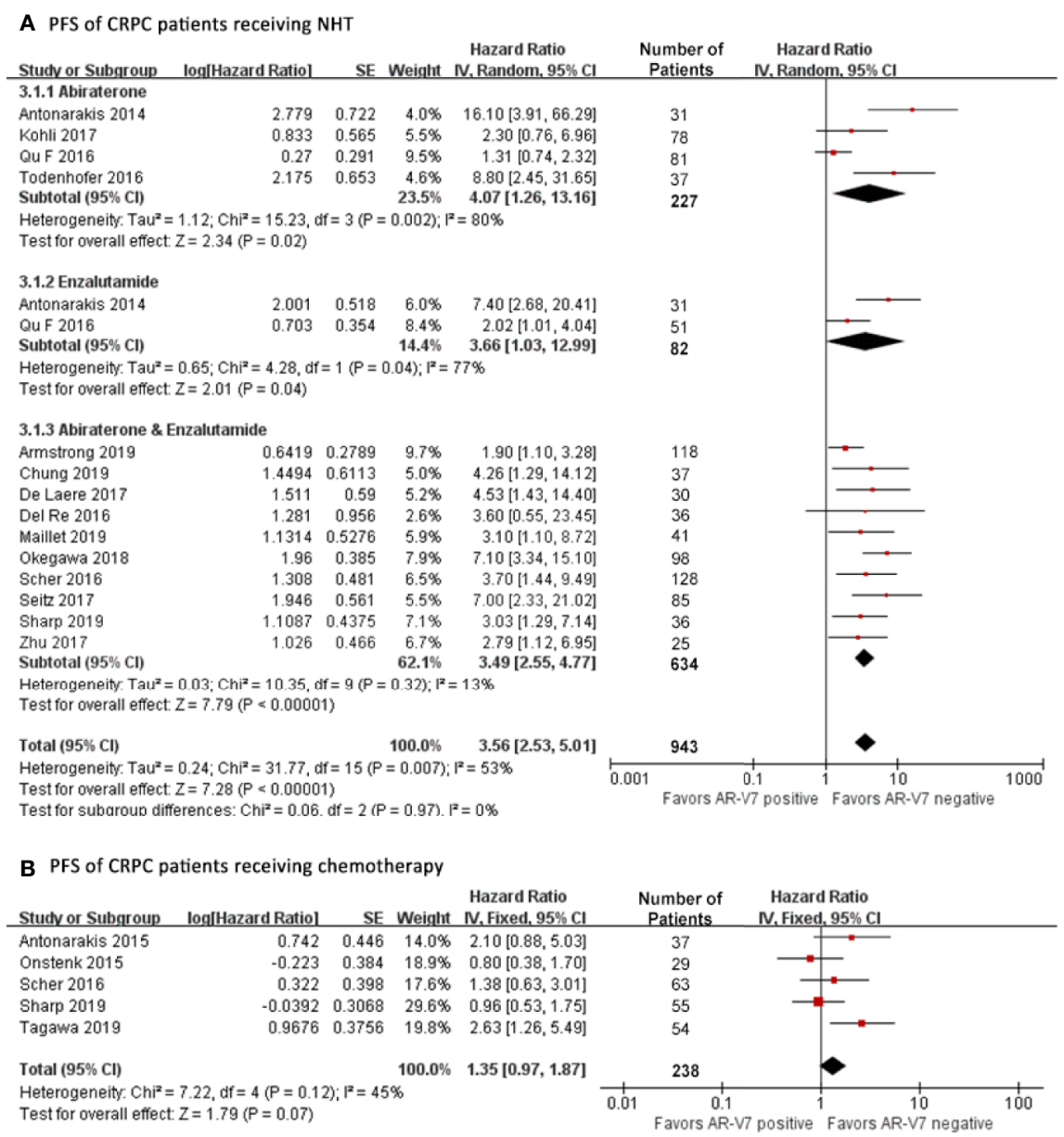

FIGURE 4 | Forest plots of hazard ratios (HRs) for PFS of NHT and chemotherapy in CRPC patients. Pooled HRs were calculated using random effect for NHT and fixed effect model for chemotherapy. The bars indicate $95 \%$ Cls. (A) PFS of CRPC patients receiving NHT. (B) PFS of CRPC patients receiving chemotherapy. NHT, novel hormonal therapy; AR-V7, androgen receptor splicing variant 7; Cl, confidence interval; PFS, progression free survival. 


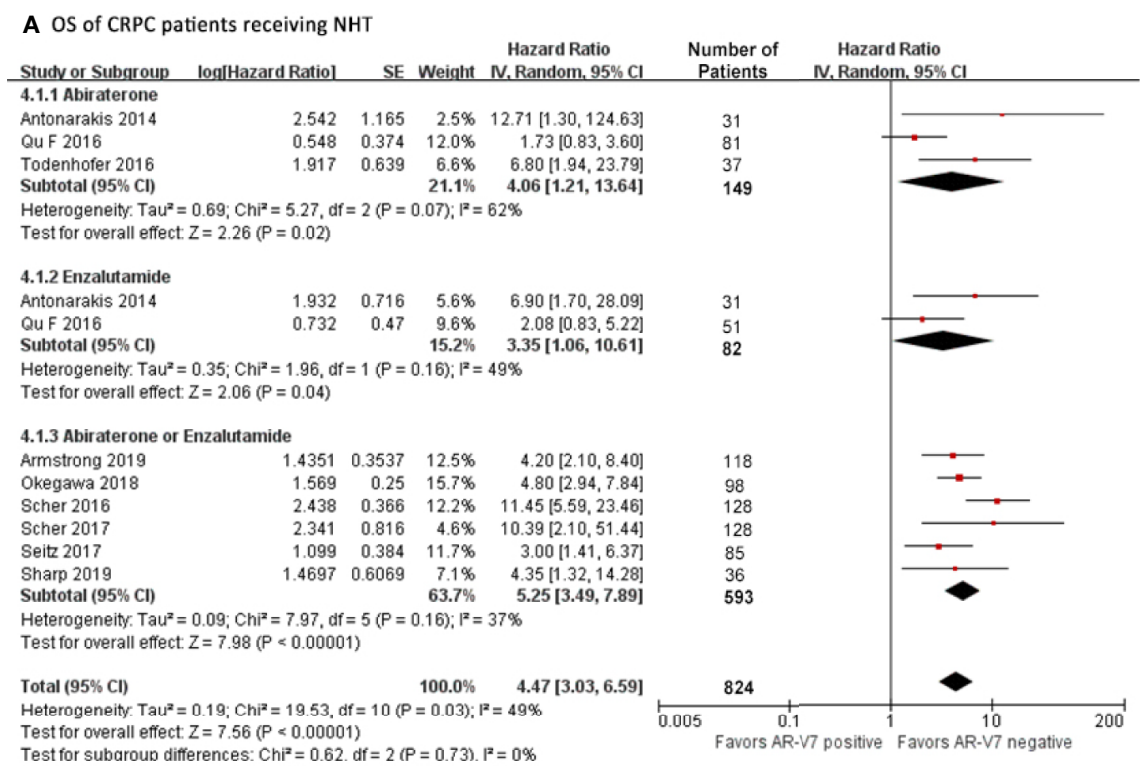

B OS of CRPC patients receiving chemotherapy
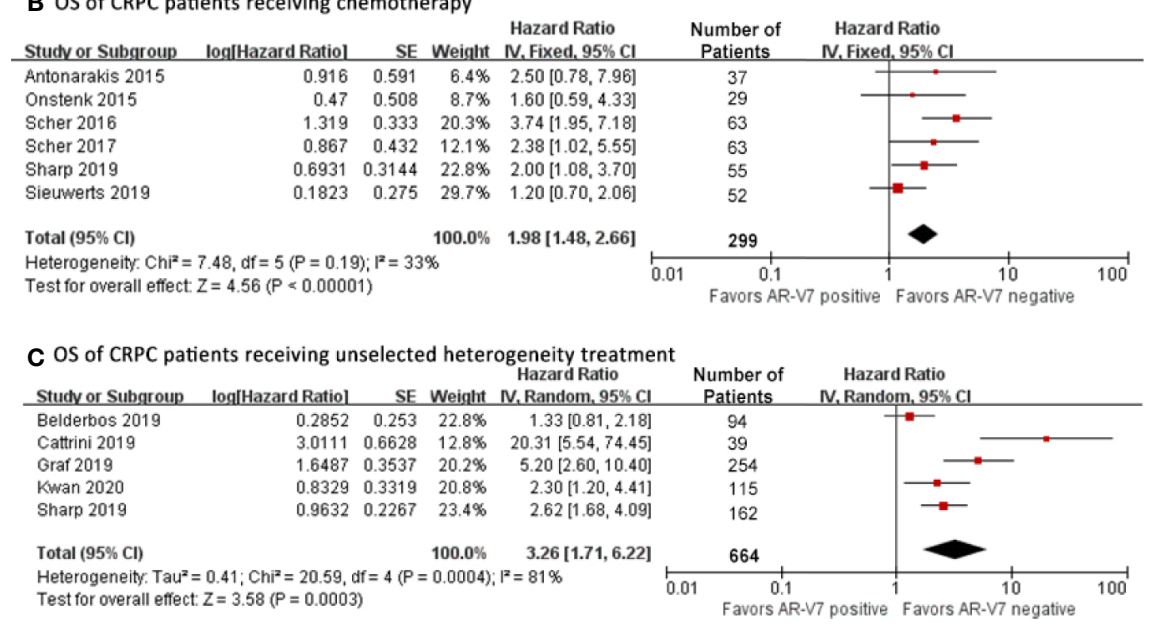

FIGURE 5 | Forest plots of hazard ratios for OS in CRPC patients received NHT, chemotherapy or non-standardized treatment. Pooled HRs were calculated using random effect model. The bars indicate 95\% Cls. (A) OS of CRPC patients receiving NHT. (B) OS of CRPC patients receiving chemotherapy. (C) OS of CRPC patients receiving non-standardized treatment. AR-V7, androgen receptor splicing variant 7; Cl, confidence interval; OS, overall survival.

\section{DISCUSSION}

This meta-analysis aimed to explore the prognosis value of ARV7 in CRPC patients treated with NHT or chemotherapy. Several reports have indicated that AR-V7 can potentially induce and promote NHT resistance in CRPC and predict the prognosis of CRPC $(6,46,47)$. It has been shown that taxane can maintain tubulin stability, inhibit cell mitosis and thereby induce apoptosis by promoting tubulin polymerization, decreasing AR nuclear localization, and inhibit depolymerization (15). Androgen receptor variants contain DNA binding domain and the hinge region. They can decrease taxane sensitivity by altering the inhibit ability of taxane of AR nuclear localization. Unlike ARv567, ARV7 lacks the hinge region, thus cannot co-sediment with microtubules or coprecipitate with dynein motor protein, it is therefore unaffected by taxane treatment (48). Although ARV7 contributes to NHT resistance and chemotherapy, its predictive role in CRPC treatment has not been validated.

Our results show that the conversion of AR-V7 positivity increased in CRPC patients after NHT but decreased after chemotherapy. The change in AR-V7 expression was first reported by Antonarakis et al. in NHT-treated CRPC patients detected by circulating tumor cell (CTC) mRNA (7). Clinical reports have indicated that the AR-V7 positivity is elevated after NHT $(8,9,17,18,20)$. A similar observation has been reported after taxane treatment, but not as statistically significant as NHT $(8,18,21)$. The present study shows that taxane chemotherapy decreased AR-V7 expression. 
The different conversion of AR-V7 expression after NHT and chemotherapy indicates different treatment mechanisms. These findings show that taxane-based therapeutic combination may be effective against drug resistance in CRPC (49), particularly for AR-V7 positive patients. However, due to the various chemotherapeutic agents and the different AR-V7 detecting assays among studies, these results need to be verified. Therefore, further research is needed to elucidate the conversion mechanism of AR-V7 expression during treatment.

Further analysis showed that, among patients who received NHT, the PSA-response proportion was lower in AR-V7positive patients than negative. This indicated that AR-V7 could potentially predict NHT resistance. In our previous study, taxane-based chemotherapy tended to be highly beneficial than NHT in AR-V7 positive patients whereas the efficacy of NHT and taxane in AR-V7 negative patients was similar (12). Recent trials reported that AR-V7-positive CRPC patients show a lower PSA response to chemotherapy than AR-V7-negative patients $(15,21,23,35)$ whereas others have reported opposite results $(38,39)$. A previous study demonstrated that AR-V7 derived from CTC was not associated with primary resistance to taxane-based chemotherapy in patients with metastatic CRPC (8). By contrast, our results indicate a significantly lower PSA response to chemotherapy in AR-V7-positive patients. The priortreatment of enrolled patients was diversiform, and the effect of prior NHT on the following chemotherapy was unclear. Further research is required to elucidate the relationship between AR-V7 and taxane chemotherapy resistance.

Sub-analysis was carried out to delineate the association of AR-V7 expression with the PFS and OS. The NHT group was further sub-classified into Abiraterone, Enzalutamide, or a combination of both. Of all patients receiving NHT, AR-V7positive patients had worse PFS and OS than AR-V7-negative patients. Since the first report by Antonarakis et al. that AR-V7 in CTCs was associated with NHT resistance and poor survival in CRPC patients $(7,8,11)$, the prognostic value of AR-V7 of CRPC patients receiving NHT has been extensively studied. Additionally, a large prospective study by Antonarakis et al. examined the negative prognostic effect of CTC-based AR-V7 testing in patients with CRPC receiving Abiraterone or Enzalutamide (9). Although AR-V7 showed its potential clinical utility as a prognostic biomarker in CRPC, the diverse findings on the predictive value and specificity of different ARV7 detection methods limited its clinical validation. Discordant CTC AR-V7 results and AR-V7 protein expression in matched, same-patient biopsies were reported by Sharp et al. whereby ARV7 positivity was associated with higher CTC counts which may have confounded outcome analyses (23). The prognostic role of AR-V7 in CRPC patients receiving chemotherapy has been a subject of research. Previous trials indicated that AR-V7 in CTCs from mCRPC was not associated with primary resistance to taxane chemotherapy and potentially responded better to taxane than NHT $(8,50)$. By contrast, some studies reported that the AR-V7 did not affect the outcome of taxane treatment in patients with mCRPC $(15,21)$. In this study, a worse outcome was observed in AR-V7 positive patients receiving chemotherapy with a nearly statistically significant PFS (HR 1.35, 95\% CI $0.97-$ $1.87, \mathrm{P}=0.07)$ and remarkably shorter OS (HR 1.98, 95\% CI 1.48$2.66, \mathrm{P}<0.001)$. Given the inconsistent results from previous studies, whether AR-V7 could be applied to guide treatment decisions in CRPC is unclear. A report by Graf et al. indicated that doctors prefer taxane to NHT for patients with a highly aggressive disease or who have received NHT previously, which might result in treatment selection tendency. After adjusting physician propensity, no marked differences in OS were observed between taxane- and NHT-treated patients (42). Elsewhere, nuclear CTC expression of AR-V7 protein was associated with better survival in $\mathrm{MCRPC}$ patients receiving taxane therapy than those receiving NHT (11). Therefore, multiple therapeutic modalities may be applied simultaneously to effectively reverse resistance in AR-V7 positive CRPC (19). Moreover, AR-V7 should be consistently monitored during treatment and more effective target agents for AR-V7 are needed. Further prospective validation studies are required to determine the prognostic role of AR-V7 in CRPC (51).

This study is limited by the different comprised sample sizes ranging from 14 to 277 patients, and the statistical power is restricted by the following: First, small sample size and limited follow-up time of some studies would yield less reliable results due to the size effect. The risk of publication bias among the studies cannot be ignored. We plotted low statistical power trials in Supplementary Figures S1-S5 by asymmetrical distribution of funnel plots for further investigation. Second, research designs of the included studies are not unified. Most trials are singlecentered and thus selection bias cannot be ruled out. Patient selection criteria are highly variable among different studies. Third, different assays were used to measure AR-V7 expression ranging from qRT-PCR, immunohistochemistry (IHC), fluorescence in-situ hybridization (FISH) and RNA in-situ hybridization (RISH). These detection methods carry different advantages and disadvantages as described in previous reviews $(51,52)$. Therefore, positivity rate may vary across studies because of different cutoff values. The sensitivity and specificity of tissue-based detection are not optimal because of the nonspecific detection of nuclear AR-V7 pre-mRNA by RISH and the nonspecific binding reaction of AR-V7 anti-body. Moreover, although it is feasible to measure AR-V7 and other AR aberrations using blood-based assays, it is still necessary to warrant the clinical validation of individual and integrated assays in the future (53). For CTC mRNA detection, CTC AR-V7 status was discordant between CellSearch and AdnaTest at low AR-V7 mRNA expression level which may confound outcome analyses (23). The sample type and AR-V7 test methods applied in the enrolled studies were detailed in Supplementary Table S1. Fourth, patients' characteristics such as tumor stage, metastasis and endpoint definitions are different. This may result in a possible discrepancy among studies (Supplementary Table S2). Last but not the least, physicians tended to choose chemotherapy rather than NHT for patients with more advanced disease or who had already received NHT as immediate prior therapy, and AR-V7 positivity was associated 
with highly aggressive prostate cancer and tumor burden: this may confound the results and generate bias. Therefore, AR-V7 was not extensively and sufficiently studied in prostate cancer.

We attempted to minimize these limitations. First, we performed a comprehensive systematic literature search on major online databases with a reproducible strategy to minimize publication bias. Second, although selection bias could not be eliminated, strict inclusion criteria were applied to minimize bias caused by different AR-V7 test assays, treatment response definition, and therapy history. Third, a subgroup analysis was performed according to different therapies and it was reported that the correlation between ARV7 and therapy outcomes was consistent across the subgroup stratified by NHT and chemotherapy. Fourth, the type of trial, tumor stage, AR-V7 test assay, therapy strategy, prior-treatment PSA, and surveillance time are detailed in tables for further analysis and reference. Biomarkers that provide accurate prognostic information for CRPC are urgently required. Several randomized clinical trials (RCTs) have indicated the benefits of early inclusion of docetaxel to androgen deprivation therapy in hormone-sensitive metastatic prostate cancer (54-57). Besides, in mCRPC, clinical data favored taxane over NHT in the second-line treatment setting for the optimal patient benefit (58). Recent research reported that novel taxane-based combination therapies potentially improve outcomes (49). However, validated biomarkers are desired for selecting suitable patients due to the risk of enhanced toxicity. Various prognostic markers have been identified for CRPC (59) but none can be applied in selecting a treatment strategy. This meta-analysis suggested that AR-V7 may be a potential therapeutic target and prognostic biomarker in CRPC patients. There is an urgent need for more prospective trials to verify the utility of AR-V7 as a biomarker in CRPC. Additionally, researches on biomarker-driven or biomarkerstratified clinical trials are required to improve AR-V7 evaluation following the findings from this meta-analysis.

\section{CONCLUSION}

The significant prognostic value of AR-V7 in CRPC patients treated with NHT or chemotherapy was determined in this metaanalysis. The AR-V7 expression significantly increased after NHT but remarkably decreased after chemotherapy. Moreover,

\section{REFERENCES}

1. Beer TM, Armstrong AJ, Rathkopf DE, Loriot Y, Sternberg CN, Higano CS, et al. Enzalutamide in metastatic prostate cancer before chemotherapy. New Engl J Med (2014) 371(5):424-33. doi: 10.1056/ NEJMoa 1405095

2. Ryan CJ, Smith MR, de Bono JS, Molina A, Logothetis CJ, de Souza P, et al. Abiraterone in metastatic prostate cancer without previous chemotherapy. New Engl J Med (2013) 368(2):138-48. doi: 10.1056/ NEJMoa 1209096

3. Ryan CJ, Smith MR, Fizazi K, Saad F, Mulders PF, Sternberg CN, et al. Abiraterone acetate plus prednisone versus placebo plus prednisone in chemotherapy-naive men with metastatic castration-resistant prostate cancer (COU-AA-302): final overall survival analysis of a randomised,
AR-V7 positive CRPC patients portend worse prognosis of NHT with a lower therapy response, poorer PFS, and OS. Besides, for chemotherapy, AR-V7 positive patients acquired fewer benefits of prognosis with a lower PSA response, shorter OS, and a nearly significantly worse PFS. Based on these results, AR-V7-positive CRPC should be considered as a specific subtype with a worse prognosis and be treated with a highly active anti-tumor therapy. Furthermore, the AR-V7 status should be consistently monitored and AR-V7 targeted therapy strategies are needed. However, cross-institutional studies are still needed to validate AR-V7 as a selection treatment marker. Future studies should aim to improve AR-V7 detection to validate the clinical utility in CRPC.

\section{DATA AVAILABILITY STATEMENT}

All datasets presented in this study are included in the article/ Supplementary Material.

\section{AUTHOR CONTRIBUTIONS}

ZW: project development, data collection, data analysis, manuscript writing. HS: data collection, data analysis, manuscript writing. NM: data collection, data analysis. QL: data collection, data analysis. YM: data collection, data analysis. CW: project development, manuscript editing. LX: project development, manuscript editing. All authors contributed to the article and approved the submitted version.

\section{ACKNOWLEDGMENTS}

We thank all researchers and participants of included studies for their contributions.

\section{SUPPLEMENTARY MATERIAL}

The Supplementary Material for this article can be found online at: https://www.frontiersin.org/articles/10.3389/fonc.2020.572590/ full\#supplementary-material

double-blind, placebo-controlled phase 3 study. Lancet Oncol (2015) 16 (2):152-60. doi: 10.1016/s1470-2045(14)71205-7

4. Beer TM, Armstrong AJ, Rathkopf D, Loriot Y, Sternberg CN, Higano CS, et al. Enzalutamide in Men with Chemotherapy-naive Metastatic Castrationresistant Prostate Cancer: Extended Analysis of the Phase 3 PREVAIL Study. Eur Urol (2017) 71(2):151-4. doi: 10.1016/j.eururo.2016.07.032

5. Li Y, Chan SC, Brand LJ, Hwang TH, Silverstein KA, Dehm SM. Androgen receptor splice variants mediate enzalutamide resistance in castrationresistant prostate cancer cell lines. Cancer Res (2013) 73(2):483-9. doi: 10.1158/0008-5472.can-12-3630

6. Hu R, Dunn TA, Wei S, Isharwal S, Veltri RW, Humphreys E, et al. Ligandindependent androgen receptor variants derived from splicing of cryptic exons signify hormone-refractory prostate cancer. Cancer Res (2009) 69 (1):16-22. doi: 10.1158/0008-5472.can-08-2764 
7. Antonarakis ES, Lu C, Wang H, Luber B, Nakazawa M, Roeser JC, et al. ARV7 and resistance to enzalutamide and abiraterone in prostate cancer. New Engl J Med (2014) 371(11):1028-38. doi: 10.1056/NEJMoa1315815

8. Antonarakis ES, Lu C, Luber B, Wang H, Chen Y, Nakazawa M, et al. Androgen Receptor Splice Variant 7 and Efficacy of Taxane Chemotherapy in Patients With Metastatic Castration-Resistant Prostate Cancer. JAMA Oncol (2015) 1(5):582-91. doi: 10.1001/jamaoncol.2015.1341

9. Antonarakis ES, Lu C, Luber B, Wang H, Chen Y, Zhu Y, et al. Clinical Significance of Androgen Receptor Splice Variant-7 mRNA Detection in Circulating Tumor Cells of Men With Metastatic Castration-Resistant Prostate Cancer Treated With First- and Second-Line Abiraterone and Enzalutamide. J Clin Oncol Off J Am Soc Clin Oncol (2017) JCO2016701961 35(19):2149-56. doi: 10.1200/jco.2016.70.1961

10. Scher HI, Graf RP, Schreiber NA, Jayaram A, Winquist E, McLaughlin B, et al. Assessment of the Validity of Nuclear-Localized Androgen Receptor Splice Variant 7 in Circulating Tumor Cells as a Predictive Biomarker for Castration-Resistant Prostate Cancer. JAMA Oncol (2018) 4(9):1179-86. doi: 10.1001/jamaoncol.2018.1621

11. Scher HI, Lu D, Schreiber NA, Louw J, Graf RP, Vargas HA, et al. Association of AR-V7 on Circulating Tumor Cells as a Treatment-Specific Biomarker With Outcomes and Survival in Castration-Resistant Prostate Cancer. JAMA Oncol (2016) 2(11):1441-9. doi: 10.1001/jamaoncol.2016.1828

12. Li H, Wang Z, Tang K, Zhou H, Liu H, Yan L, et al. Prognostic Value of Androgen Receptor Splice Variant 7 in the Treatment of Castration-resistant Prostate Cancer with Next generation Androgen Receptor Signal Inhibition: A Systematic Review and Meta-analysis. Eur Urol Focus (2017) 4(4):529-39. doi: 10.1016/j.euf.2017.01.004

13. Takeuchi T, Okuno Y, Hattori-Kato M, Zaitsu M, Mikami K. Detection of ARV7 mRNA in whole blood may not predict the effectiveness of novel endocrine drugs for castration-resistant prostate cancer. Res Rep Urol (2016) 8:21-5. doi: $10.2147 /$ rru.s98877

14. To SQ, Kwan EM, Fettke HC, Mant A, Docanto MM, Martelotto L, et al. Expression of Androgen Receptor Splice Variant 7 or 9 in Whole Blood Does Not Predict Response to Androgen-Axis-targeting Agents in Metastatic Castration-resistant Prostate Cancer. Eur Urol (2018) 73(6):818-21. doi: 10.1016/j.eururo.2018.01.007

15. Tagawa ST, Antonarakis ES, Gjyrezi A, Galletti G, Kim S, Worroll D, et al. Expression of AR-V7 and ARv(567es) in Circulating Tumor Cells Correlates with Outcomes to Taxane Therapy in Men with Metastatic Prostate Cancer Treated in TAXYNERGY. Clin Cancer Res an Off J Am Assoc Cancer Res (2019) 25(6):1880-8. doi: 10.1158/1078-0432.ccr-18-0320

16. Antonarakis ES, Scher HI. Do Patients With AR-V7-Positive Prostate Cancer Benefit from Novel Hormonal Therapies? It All Depends on Definitions. Eur Urol (2017) 71(1):4-6. doi: 10.1016/j.eururo.2016.08.038

17. Armstrong AJ, Halabi S, Luo J, Nanus DM, Giannakakou P, Szmulewitz RZ, et al. Prospective Multicenter Validation of Androgen Receptor Splice Variant 7 and Hormone Therapy Resistance in High-Risk Castration-Resistant Prostate Cancer: The PROPHECY Study. J Clin Oncol Off J Am Soc Clin Oncol (2019) 37(13):1120-9. doi: 10.1200/jco.18.01731

18. Nakazawa M, Lu C, Chen Y, Paller CJ, Carducci MA, Eisenberger MA, et al. Serial blood-based analysis of AR-V7 in men with advanced prostate cancer. Ann Oncol Off J Eur Soc Med Oncol / ESMO (2015) 26(9):1859-65. doi: $10.1093 /$ annonc/mdv282

19. Sharp A, Coleman I, Yuan W, Sprenger C, Dolling D, Rodrigues DN, et al. Androgen receptor splice variant-7 expression emerges with castration resistance in prostate cancer. J Clin Invest (2019) 129(1):192-208. doi: 10.1172/jci122819

20. Welti J, Rodrigues DN, Sharp A, Sun S, Lorente D, Riisnaes R, et al. Analytical Validation and Clinical Qualification of a New Immunohistochemical Assay for Androgen Receptor Splice Variant-7 Protein Expression in Metastatic Castration-resistant Prostate Cancer. Eur Urol (2016) 70(4):599-608. doi: 10.1016/j.eururo.2016.03.049

21. Sieuwerts AM, Onstenk W, Kraan J, Beaufort CM, Van M, De Laere B, et al. AR splice variants in circulating tumor cells of patients with castrationresistant prostate cancer: relation with outcome to cabazitaxel. Mol Oncol (2019) 13(8):1795-807. doi: 10.1002/1878-0261.12529

22. Zadra G, Ribeiro CF, Chetta P, Ho Y, Cacciatore S, Gao X, et al. Inhibition of de novo lipogenesis targets androgen receptor signaling in castration-resistant prostate cancer. Proc Natl Acad Sci USA (2019) 116:631-40.
23. Sharp A, Welti JC, Lambros MBK, Dolling D, Rodrigues DN, Pope L, et al. Clinical Utility of Circulating Tumour Cell Androgen Receptor Splice Variant-7 Status in Metastatic Castration-resistant Prostate Cancer. Eur Urol (2019) 76(5):676-85. doi: 10.1016/j.eururo.2019.04.006

24. Zhu Y, Sharp A, Anderson CM, Silberstein JL, Taylor M, Lu C, et al. Novel Junction-specific and Quantifiable In Situ Detection of AR-V7 and its Clinical Correlates in Metastatic Castration-resistant Prostate Cancer. Eur Urol (2018) 73:727-35.

25. Steinestel J, Luedeke M, Arndt A, Schnoeller TJ, Lennerz JK, Wurm C. Detecting predictive androgen receptor modifications in circulating prostate cancer cells. Oncotarget (2015) 10(41):4213-23.

26. Todenhofer T, Azad A, Stewart C, Gao J, Eigl BJ, Gleave ME, et al. AR-V7 transcripts in whole blood RNA of patients with metastatic castration resistant prostate cancer correlate with response to Abiraterone acetate. J Urol (2016) 197(1):135-42.

27. Qu F, Xie W, Nakabayashi M, Zhang H, Jeong SH, Wang X, et al. Association of AR-V7 and prostate specific antigen RNA levels in blood with efficacy of abiraterone acetate and enzalutamide treatment in men with prostate cancer. Clin Cancer Research: an Off J Am Assoc Cancer Res (2016) 23(3):726-34.

28. Del Re M, Biasco E, Crucitta S, Derosa L, Rofi E, Orlandini C, et al. The Detection of Androgen Receptor Splice Variant 7 in Plasma-derived Exosomal RNA Strongly Predicts Resistance to Hormonal Therapy in Metastatic Prostate Cancer Patients. Eur Urol (2017) 71:680-7.

29. Seitz AK, Thoene S, Bietenbeck A, Nawroth R, Tauber R, Thalgott M, Schmid S, et al. AR-V7 in Peripheral Whole Blood of Patients with Castrationresistant Prostate Cancer: Association with Treatment-specific Outcome Under Abiraterone and Enzalutamide. Eur Urol (2017) 72(5):828-34.

30. De Laere B, van Dam PJ, Whitington T, Mayrhofer M, Diaz EH, Van den EG, et al. Comprehensive Profiling of the Androgen Receptor in Liquid Biopsies from Castration-resistant Prostate Cancer Reveals Novel Intra-AR Structural Variation and Splice Variant Expression Patterns. Eur Urol (2017) 72(2):192-200.

31. Scher HI, Graf RP, Schreiber NA, McLaughlin B, Lu D, Louw J, et al. Nuclearspecific AR-V7 Protein Localization is Necessary to Guide Treatment Selection in Metastatic Castration-resistant Prostate Cancer. Eur Urol (2017) 71:874-82.

32. Okegawa T, Ninomiya N, Masuda K, Nakamura Y, Tambo M, Nutahara K. AR-V7 in circulating tumor cells cluster as a predictive biomarker of abiraterone acetate and enzalutamide treatment in castration-resistant prostate cancer patients. Prostate (2018) 78:576-82.

33. Kohli M, Ho Y, Hillman DW, Van Etten JL, Henzler C, Yang R, et al. Androgen Receptor Variant AR-V9 Is Coexpressed with AR-V7 in Prostate Cancer Metastases and Predicts Abiraterone Resistance. Clin Cancer Research: an Off J Am Assoc Cancer Res (2017) 23:4704-15.

34. Tommasi S, Pilato B, Carella C, Lasorella A, Danza K, Vallini I, et al. Standardization of CTC AR-V7 PCR assay and evaluation of its role in castration resistant prostate cancer progression. Prostate (2018) 79(1):54-61.

35. Cattrini C, Rubagotti A, Zinoli L, Cerbone L, Zanardi E, Capaia M, et al. Role of Circulating Tumor Cells (CTC), Androgen Receptor Full Length (AR-FL) and Androgen Receptor Splice Variant 7 (AR-V7) in a Prospective Cohort of Castration-Resistant Metastatic Prostate Cancer Patients. Cancers (Basel) (2019) 11(9):1365-74. doi: 10.3390/cancers11091365

36. Chung JS, Wang Y, Henderson J, Singhal U, Qiao Y, Zaslavsky AB, et al. Circulating Tumor Cell-Based Molecular Classifier for Predicting Resistance to Abiraterone and Enzalutamide in Metastatic Castration-Resistant Prostate Cancer. Neoplasia (New York NY) (2019) 21:802-9.

37. Del Re M, Crucitta S, Sbrana A, Rofi E, Paolieri F, Gianfilippo G, et al. AR-V7 and AR-FL expression is associated with clinical outcome: a translational study in patients with castrate resistant prostate cancer. BJU Int (2019) 124:693-700.

38. El-Heliebi A, Hille C, Laxman N, Svedlund J, Haudum C, Ercan E, et al. In Situ Detection and Quantification of AR-V7, AR-FL, PSA, and KRAS Point Mutations in Circulating Tumor Cells. Clin Chem (2018) 64(3):536-46. doi: 10.1373/clinchem.2017.281295

39. Worroll D, Galletti G, Gjyrezi A, Nanus DM, Tagawa ST, Giannakakou P. Androgen receptor nuclear localization correlates with AR-V7 mRNA expression in circulating tumor cells (CTCs) from metastatic castration resistance prostate cancer patients. Phys Biol (2019) 16(3):036003. doi: 10.1088/1478-3975/ab073a

40. Belderbos BPS, Sieuwerts AM, Hoop EO, Mostert B, Kraan J, Hamberg P, et al. Associations between AR-V7 status in circulating tumour cells, circulating 
tumour cell count and survival in men with metastatic castration-resistant prostate cancer. Eur J Cancer (Oxford England: 1990) (2019) 121:48-54.

41. Maillet D, Allioli N, Peron J, Plesa A, Decaussin-Petrucci M, Tartas S, et al. Improved Androgen Receptor Splice Variant 7 Detection Using a Highly Sensitive Assay to Predict Resistance to Abiraterone or Enzalutamide in Metastatic Prostate Cancer Patients. Eur Urol Oncol (2019) S2588-9311(19) 30136-1.

42. Graf RP, Hullings M, Barnett ES, Carbone E, Dittamore R, Scher HI. Clinical Utility of the Nuclear-localized AR-V7 Biomarker in Circulating Tumor Cells in Improving Physician Treatment Choice in Castration-resistant Prostate Cancer. Eur Urol (2019) 77(2):170-7. doi: 10.1016/j.eururo.2019.08.020

43. Erb HHH, Sparwasser P, Diehl T, Hemmerlein-Thomas M, Tsaur I, Jüngel E, et al. AR-V7 Protein Expression in Circulating Tumour Cells Is Not Predictive of Treatment Response in mCRPC. Urologia Internationalis (2020) 17:1-10.

44. Kwan EM, Fettke H, Docanto MM, To SQ, Bukczynska P, Mant A, et al. Prognostic Utility of a Whole-blood Androgen Receptor-based Gene Signature in Metastatic Castration-resistant Prostate Cancer. Eur Urol Focus (2019).

45. Liberati A, Altman DG, Tetzlaff J, Mulrow C, Gotzsche PC, Ioannidis JP, et al. The PRISMA statement for reporting systematic reviews and meta-analyses of studies that evaluate health care interventions: explanation and elaboration. PloS Med (2009) 6(7):e1000100. doi: 10.1371/journal.pmed.1000100

46. Guo Z, Yang X, Sun F, Jiang R, Linn DE, Chen H, et al. A novel androgen receptor splice variant is up-regulated during prostate cancer progression and promotes androgen depletion-resistant growth. Cancer Res (2009) 69 (6):2305-13. doi: Analytical Validation and Clinical Qualification of a New Immunohistochemical Assay for Androgen Receptor Splice Variant-7 Protein Expression in Metastatic Castration-resistant Prostate Cancer10.1158/00085472.can-08-3795.

47. Dehm SM, Schmidt LJ, Heemers HV, Vessella RL, Tindall DJ. Splicing of a novel androgen receptor exon generates a constitutively active androgen receptor that mediates prostate cancer therapy resistance. Cancer Res (2008) 68(13):5469-77. doi: 10.1158/0008-5472.can-08-0594

48. Thadani-Mulero M, Portella L, Sun S, Sung M, Matov A, Vessella RL, et al. Androgen receptor splice variants determine taxane sensitivity in prostate cancer. Cancer Res (2014) 74(8):2270-82. doi: 10.1158/0008-5472.can-13-2876

49. Corn PG, Agarwal N, Araujo JC, Sonpavde G. Taxane-based Combination Therapies for Metastatic Prostate Cancer. Eur Urol Focus (2017) 5(3):369-80. doi: 10.1016/j.euf.2017.11.009

50. Onstenk W, Sieuwerts AM, Kraan J, Van M, Nieuweboer AJ, Mathijssen RH, et al. Efficacy of Cabazitaxel in Castration-resistant Prostate Cancer Is Independent of the Presence of AR-V7 in Circulating Tumor Cells. Eur Urol (2015) 68(6):939-45. doi: 10.1016/j.eururo.2015.07.007

51. Bastos DA, Antonarakis ES. CTC-derived AR-V7 detection as a prognostic and predictive biomarker in advanced prostate cancer. Expert Rev Mol Diagn (2018) 18(2):155-63. doi: 10.1080/14737159.2018.1427068
52. Luo J. Development of AR-V7 as a putative treatment selection marker for metastatic castration-resistant prostate cancer. Asian J Androl (2016) 18 (4):580. doi: 10.4103/1008-682x.178490

53. Luo J, Attard G, Balk SP, Bevan C, Burnstein K, Cato L, et al. Role of Androgen Receptor Variants in Prostate Cancer: Report from the 2017 Mission Androgen Receptor Variants Meeting. Eur Urol (2018) 73(5):715-23. doi: 10.1016/j.eururo.2017.11.038

54. Gravis G, Boher JM, Joly F, Soulie M, Albiges L, Priou F, et al. Androgen Deprivation Therapy (ADT) Plus Docetaxel Versus ADT Alone in Metastatic Non castrate Prostate Cancer: Impact of Metastatic Burden and Long-term Survival Analysis of the Randomized Phase 3 GETUG-AFU15 Trial. Eur Urol (2016) 70(2):256-62. doi: 10.1016/j.eururo.2015.11.005

55. James ND, Sydes MR, Clarke NW, Mason MD, Dearnaley DP, Spears MR, et al. Addition of docetaxel, zoledronic acid, or both to first-line long-term hormone therapy in prostate cancer (STAMPEDE): survival results from an adaptive, multiarm, multistage, platform randomised controlled trial. Lancet (2016) 387(10024):1163-77. doi: 10.1016/s0140-6736(15)01037-5

56. Gravis G, Fizazi K, Joly F, Oudard S, Priou F, Esterni B, et al. Androgendeprivation therapy alone or with docetaxel in non-castrate metastatic prostate cancer (GETUG-AFU 15): a randomised, open-label, phase 3 trial. Lancet Oncol (2013) 14(2):149-58. doi: 10.1016/S1470-2045(12) 70560-0

57. Sweeney CJ, Chen YH, Carducci M, Liu G, Jarrard DF, Eisenberger M, et al. Chemohormonal Therapy in Metastatic Hormone-Sensitive Prostate Cancer. New Engl J Med (2015) 373(8):737-46. doi: 10.1056/NEJMoa1503747

58. Oh WK, Miao R, Vekeman F, Sung J, Cheng WY, Gauthier-Loiselle M, et al. Real-world Characteristics and Outcomes of Patients With Metastatic Castration-resistant Prostate Cancer Receiving Chemotherapy Versus Androgen Receptor-targeted Therapy After Failure of First-line Androgen Receptor-targeted Therapy in the Community Setting. Clin Genitourinary Cancer (2017) S1558-7673(17)30170-2: 10.1016/j.clgc.2017.06.004

59. Cucchiara V, Cooperberg MR, Dall'Era M, Lin DW, Montorsi F, Schalken JA, et al. Genomic Markers in Prostate Cancer Decision Making. Eur Urol (2018) 73(4):572-82. doi: 10.1016/j.eururo.2017.10.036

Conflict of Interest: The authors declare that the research was conducted in the absence of any commercial or financial relationships that could be construed as a potential conflict of interest.

Copyright $\odot 2020$ Wang, Shen, Ma, Li, Mao, Wang and Xie. This is an open-access article distributed under the terms of the Creative Commons Attribution License (CC BY). The use, distribution or reproduction in other forums is permitted, provided the original author(s) and the copyright owner(s) are credited and that the original publication in this journal is cited, in accordance with accepted academic practice. No use, distribution or reproduction is permitted which does not comply with these terms. 